\title{
New Models for Two Real Scalar Fields and Their Kink-Like Solutions
}

\author{
A. Alonso-Izquierdo, ${ }^{1}$ D. Bazeia, ${ }^{2,3,4}$ L. Losano, ${ }^{3,4}$ and J. Mateos Guilarte ${ }^{5}$ \\ ${ }^{1}$ Departamento de Matematica Aplicada and IUFFyM, Universidad de Salamanca, 37007 Salamanca, Spain \\ ${ }^{2}$ Instituto de Física, Universidade de São Paulo, 05314-970 São Paulo, SP, Brazil \\ ${ }^{3}$ Departamento de Física, Universidade Federal da Paraíba, 58051-970 João Pessoa, PB, Brazil \\ ${ }^{4}$ Departamento de Física, Universidade Federal de Campina Grande, 58109-970 Campina Grande, PB, Brazil \\ ${ }^{5}$ Departamento de Física Fundamental and IUFFyM, Universidad de Salamanca, 37007 Salamanca, Spain
}

Correspondence should be addressed to A. Alonso-Izquierdo; alonsoiz@usal.es

Received 10 June 2013; Accepted 7 August 2013

Academic Editor: Chao-Qiang Geng

Copyright (c) 2013 A. Alonso-Izquierdo et al. This is an open access article distributed under the Creative Commons Attribution License, which permits unrestricted use, distribution, and reproduction in any medium, provided the original work is properly cited.

We study the presence of kinks in models described by two real scalar fields in bidimensional spacetime. We generate new twofield models, constructed from distinct but important one-field models, and we solve them with techniques that we introduce in the current work. We illustrate the results with several examples of current interest to high energy physics.

\section{Introduction}

The presence of kinks and solitons in models described by real scalar fields is of direct interest to high energy physics $[1,2]$ and other areas of nonlinear science $[3,4]$. To mention specific studies, in high energy physics kinks appear in very interesting systems introduced, for instance, in $[5,6]$. In condensed matter one can investigate domain walls in magnetic systems $[7,8]$, and nonlinear excitations in BoseEinstein condensates $[9,10]$, to quote just a few examples.

In this work we focus on one-field and two-field models in $(1,1)$ spacetime dimensions. Two very interesting models described by a single real scalar field are known as the sine-Gordon and the $\phi^{4}$ models, engendering spontaneous symmetry breaking. The $\phi^{4}$ model is described by a fourthorder polynomial potential and supports kink-like solutions, whereas the sine-Gordon model is characterized by a nonpolynomial potential and supports not only solitons but also multisoliton and breather solutions. Fluctuations around the solitons and $\lambda \phi^{4}$ kinks, however, are governed by the $\ell=1$ and $\ell=2$ reflectionless Hamiltonians of a general family known from supersymmetric quantum mechanics [11]. Moreover, a rich family of nonpolynomial models with spontaneous symmetry breaking was proposed in [12]. The main feature of the family of kinks arising in this family is that the Hamiltonians governing the kink small fluctuations cover many of the remaining transparent SUSY Hamiltonians; see also [13].

We start with these one-field models, which are described by polynomial and nonpolynomial $W=W(\phi)$, and we then move on to the two-field models constructed from the previous ones. Our aim is to identify kink solutions in these new models, which in general is a very difficult endeavor, as Rajaraman [14] notices: "This already brings us to the stage where no general methods are available for obtaining all localized static solutions (kinks), given the field equations. However, some solutions, but by no means all, can be obtained for a class of such Lagrangians using a little trial and error." In this work we develop a technique which generates twocomponent kink solutions for two-field models in a straightforward while way avoiding the use of the trial and error method mentioned by Rajaraman. We mention, however, that there exist two scalar field theory models [15] and even models of three scalar field ones [16] such that all the kink solutions can be found due to the complete integrability of the analogue mechanical problem.

For simplicity, we use natural units and then redefine fields and coordinates such that fields and space and time 
are all dimensionless. The study starts in Section 2, and the one-field and two-field models are then used in Section 3 to generate new models, described by two fields. In this section we deal with polynomial potential, and so, to enlarge the scope of the work, in Section 4 we introduce another family of models, containing a nonpolynomial function of the field $\chi$. We end the work in Section 5, where we introduce some comments and conclusions.

\section{Generalities}

Let us first consider one-field models. We take the Lagrange density in the following form:

$$
\mathscr{L}=\frac{1}{2} \partial^{\mu} \phi \partial_{\mu} \phi-V(\phi) .
$$

Here we deal with topological solutions, so we write the potential $V(\phi)$ in the following form:

$$
V(\phi)=\frac{1}{2} W_{\phi}^{2}
$$

where $W=W(\phi)$, and $W_{\phi}$ stands for the derivative with respect to $\phi$; that is, $W_{\phi}=d W / d \phi$. The equation of motion for static field configuration is given by

$$
\phi^{\prime \prime}=\frac{d U}{d \phi}=W_{\phi} W_{\phi \phi} .
$$

Here we are using $\phi^{\prime}=d \phi / d x$, and so forth. The energy density for static solutions can be written as

$$
\epsilon(x)=\frac{1}{2} \phi^{\prime 2}+\frac{1}{2} W_{\phi}^{2}=\frac{1}{2}\left(\phi^{\prime}-W_{\phi}\right)^{2}+\frac{d W}{d x}
$$

for smooth superpotentials. We note that the energy is minimized to the value

$$
E_{\mathrm{BPS}}=|W(\phi(\infty))-W(\phi(-\infty))|
$$

for field configurations that obey the first-order equation

$$
\phi^{\prime}=W_{\phi}
$$

This is the Bogomol'nyi bound, and we can easily see that solutions to (6) also solve the equation of motion (3). The field configurations that solve the first-order equation are named Bogomol'nyi-Prasad-Sommerfeld (BPS) states [17, 18]. We note that since the potential does not see the sign of $W$, there are in fact two first-order equations, one for $W$ and the other with $W$ changed to $-W$. This is related to the spatial reflection symmetry $x \rightarrow-x$, which provides us with the kink/antikink solutions.

Two important models in the previous class of models are the $\phi^{4}$ model, where

$$
W(\phi)=\phi-\frac{1}{3} \phi^{3}
$$

and the sine-Gordon model, where

$$
W(\phi)=\sin (\phi) \text {. }
$$

The potentials are, respectively,

$$
\begin{aligned}
& V(\phi)=\frac{1}{2}\left(1-\phi^{2}\right)^{2}, \\
& V(\phi)=\frac{1}{2} \cos ^{2}(\phi) .
\end{aligned}
$$

These models have solutions in the following form for the $\phi^{4}$ model,

$$
\phi(x)=\tanh (x),
$$

and for the sine-Gordon model,

$$
\phi(x)=\arcsin (\tanh (x))+k \pi, \quad k=0, \pm 1, \pm 2, \ldots,
$$

where $k$ identifies one among the infinity of topological sectors of the sine-Gordon model.

Let us now consider two-field models. We start with the Lagrange density

$$
\mathscr{L}=\frac{1}{2} \partial^{\mu} \phi \partial_{\mu} \phi+\frac{1}{2} \partial^{\mu} \chi \partial_{\mu} \chi-V(\phi, \chi) .
$$

For static configurations, the equations of motion become

$$
\phi^{\prime \prime}=\frac{\partial V}{\partial \phi}, \quad \chi^{\prime \prime}=\frac{\partial V}{\partial \chi} .
$$

We suppose that the potential $V(\phi, \chi)$ is given in terms of the superpotential $W(\phi, \chi)$ by

$$
V(\phi, \chi)=\frac{1}{2} W_{\phi}^{2}+\frac{1}{2} W_{\chi}^{2}
$$

where $W_{\phi}=\partial W / \partial \phi$ and $W_{\chi}=\partial W / \partial \chi$. Notice that the critical points of the superpotential $W(\phi, \chi)$ provide us with the set of vacua $\mathscr{M}=\left\{(\phi, \chi) \in \mathbb{R}^{2}: V(\phi, \chi)=0\right\}$ for the field theory model. The energy density has the form

$$
\begin{aligned}
\epsilon(x) & =\frac{1}{2}\left(\phi^{\prime 2}+\chi^{\prime 2}+W_{\phi}^{2}+W_{\chi}^{2}\right) \\
& =\frac{1}{2}\left[\left(\phi^{\prime}-W_{\phi}\right)^{2}+\left(\chi^{\prime}-W_{\chi}\right)^{2}\right]+d W .
\end{aligned}
$$

The minimum energy solutions comply with

$$
\phi^{\prime}=W_{\phi}, \quad \chi^{\prime}=W_{\chi}
$$

leading us to the BPS energy

$$
E_{\mathrm{BPS}}=|W(\phi(\infty), \chi(\infty))-W(\phi(-\infty), \chi(-\infty))|
$$

for smooth superpotentials. In terms of the superpotential, the equations of motion for static fields are written as

$$
\begin{aligned}
& \phi^{\prime \prime}=W_{\phi} W_{\phi \phi}+W_{\chi} W_{\chi \phi}, \\
& \chi^{\prime \prime}=W_{\phi} W_{\phi \chi}+W_{\chi} W_{\chi \chi},
\end{aligned}
$$

which are solved by the first-order equations (16), for $W_{\phi \chi}=$ $W_{\chi \phi}$, as we require in this work. Solutions to these first-order 
equations are BPS states, which solve the equations of motion. The sectors where the potential has BPS states are named BPS sectors.

As an example, let us consider the model characterized by the superpotential

$$
W(\phi, \chi)=\phi-\frac{1}{3} \phi^{3}-r \phi \chi^{2}
$$

which has been studied by Shifman et al. in the context of $N=1$ supersymmetric Wess-Zumino models with two chiral superfields $[19,20]$. In the purely bosonic framework the presence of domain walls and its stability have been analyzed in the references [21-23], while in [24, 25] the complete structure of this type of solutions is given in two critical values of the coupling between the two scalar fields by exploiting the integrability of the analogue mechanical system associated with this model. This well-known model will be used in the following sections to illustrate the applicability of the novel procedure introduced in this paper which allows the identification of kink-like solutions in new field theories.

The first-order ODE (16) in this case are written as

$$
\phi^{\prime}=1-\phi^{2}-r \chi^{2}, \quad \chi^{\prime}=-2 r \phi \chi .
$$

The potential is given by

$$
V=\frac{1}{2}\left(1-\phi^{2}-r \chi^{2}\right)^{2}+2 r^{2} \phi^{2} \chi^{2}
$$

which can be seen as an extension of the $\phi^{4}$ model to the case of two fields. Here we consider $r$ to be real and positive. The vacuum set comprises four elements:

$$
\mathscr{M}=\left\{v_{1,2}=( \pm 1,0), v_{3,4}=\left(0, \pm \sqrt{\frac{1}{r}}\right)\right\} .
$$

Associated with the superpotential (19) we can find five BPS sectors (here we do not distinguish between kinks and antikinks) by analyzing the first-order ODE (20). Indeed this model is a very special case because an integrating factor can be calculated for the orbit equation extracted from (20). The kink trajectories are given by

$$
\phi^{2}+\frac{r}{1-2 r} \chi^{2}-\gamma \chi^{1 / r}=1, \quad \text { where } \gamma \in\left(-\infty, \gamma_{C}\right]
$$

with $\gamma_{C}=2 r^{(1 / 2 r)+1} /(1-2 r)$ and $r \neq 1 / 2$. For the range $\gamma \in$ $\left(-\infty, \gamma_{C}\right)$ the formula (23) describes kinks connecting the vacua $v_{3}$ and $v_{4}$ with $E_{\mathrm{BPS}}=4 / 3$ while the value $\gamma=\gamma_{\mathrm{C}}$ yields kinks linking the points $v_{1,2}$ with $v_{3,4}$ with $E_{\mathrm{BPS}}=2 / 3$. From construction all the kinks living in a specific topological sector are energy degenerate.

In particular the $\gamma=-\infty$ and $\gamma=0$ members of (23) correspond, respectively, to the one-component kink

$$
\phi(x)= \pm \tanh (x), \quad \chi(x)=0,
$$

and the two-component kink

$$
\begin{gathered}
\phi(x)= \pm \tanh (2 r x), \\
\chi(x)= \pm \sqrt{\frac{1}{r}-2} \operatorname{sech}(2 r x)
\end{gathered}
$$

lying in the topological sector connecting the minima $v_{3}$ and $v_{4}$; see Figure 1 .

As an illustrative sample we introduce a kink solution

$$
\begin{aligned}
& \phi= \pm \frac{1}{2}(1 \pm \tanh (x)), \\
& \chi= \pm \frac{1}{2}(1 \mp \tanh (x))
\end{aligned}
$$

lying in the topological sectors joining the points $v_{3,4}$ with the points $v_{1,2}$ for the case $r=1$; see Figure 2 .

We remark that different superpotentials can generate the same potential. Indeed in this model other superpotentials than (19) have been identified for several particular values of the coupling constant $r$. This fact provides us with new degenerated BPS and non-BPS solutions in the topological sector joining the points $v_{3}$ and $v_{4}$; see [24].

\section{New Models}

To generate new two-field models and the accompanying static solutions, we proceed as follows. We start from the onefield model, with the superpotential written in the form

$$
W(\phi)=\int^{\phi} f(y) d y
$$

This gives the first-order equation

$$
\phi^{\prime}=f(\phi),
$$

and for the $\phi^{4}$ and sine-Gordon models we have $f(\phi)=1-\phi^{2}$ and $f(\phi)=\cos (\phi)$, respectively.

Now, to introduce two-field models, we get inspiration from the previous model, given by (19) and we propose the following superpotential:

$$
W(\phi, \chi)=\int^{\phi} f(y) d y-r \phi \chi^{2} .
$$

This generates the field potential term

$$
V(\phi, \chi)=\frac{1}{2}\left[f(\phi)-r \chi^{2}\right]^{2}+2 r^{2} \phi^{2} \chi^{2}
$$

The critical points of the superpotential, determined by $W_{\phi}=$ $f(\phi)-r \chi^{2}=0$ and $W_{\chi}=-2 r \phi \chi=0$, provide us with the vacua of the model:

$$
\mathscr{M}=\left\{(\phi, \chi) \in \mathbb{R}^{2}:\left(\phi^{(i)}, 0\right),\left(0, \pm \sqrt{\frac{f(0)}{r}}\right)\right\},
$$

where $\phi^{(i)}, i=1, \ldots, n$, are the roots of $f(\phi)$. Therefore this kind of models involves $n+2$ vacua assuming that $f(0) \neq 0$. The static solutions are obtained from the first-order equations

$$
\phi^{\prime}=f(\phi)-r \chi^{2}, \quad \chi^{\prime}=-2 r \phi \chi
$$




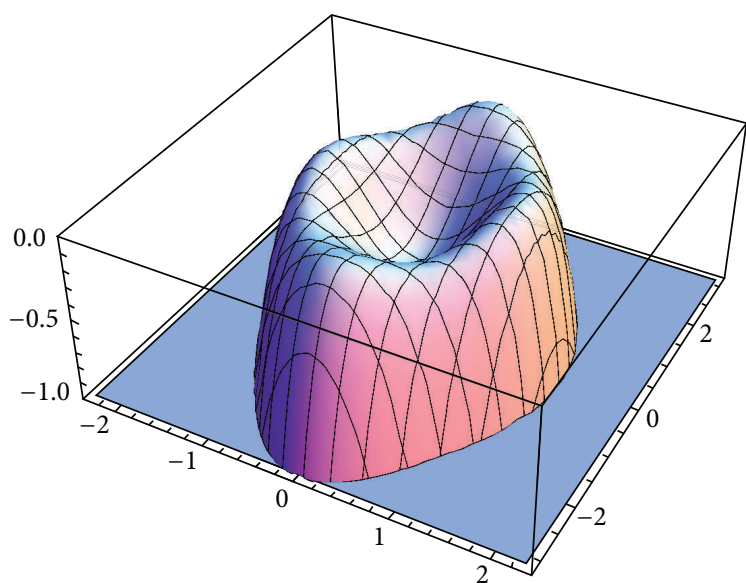

(a)

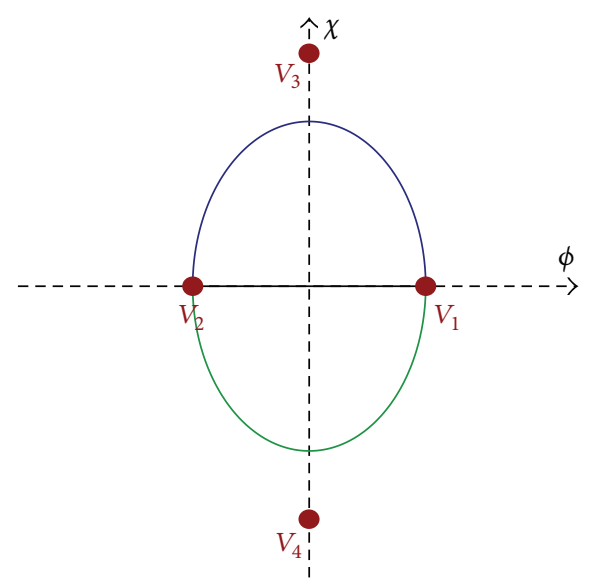

(b)

FIGURE 1: Graphics of the potential $V(\phi, \chi)$ with $r=1 / 4$ and orbits of the kinks (24) and (25) in the internal plane.

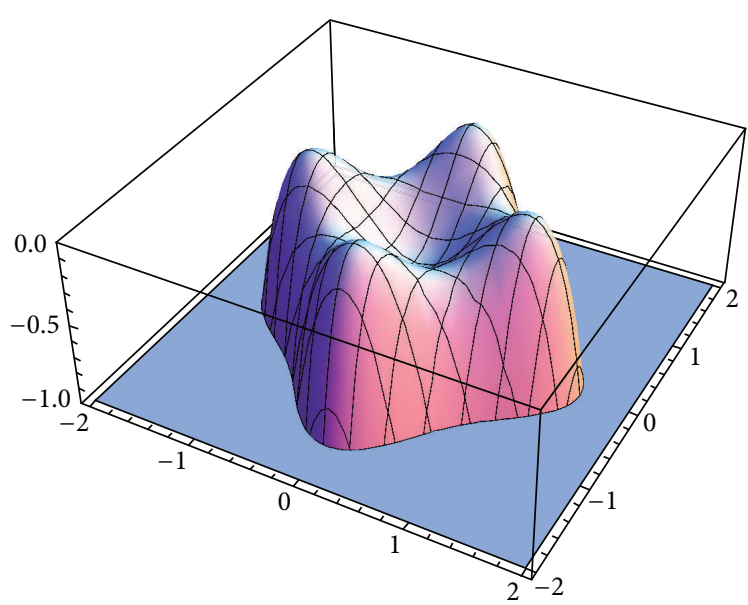

(a)

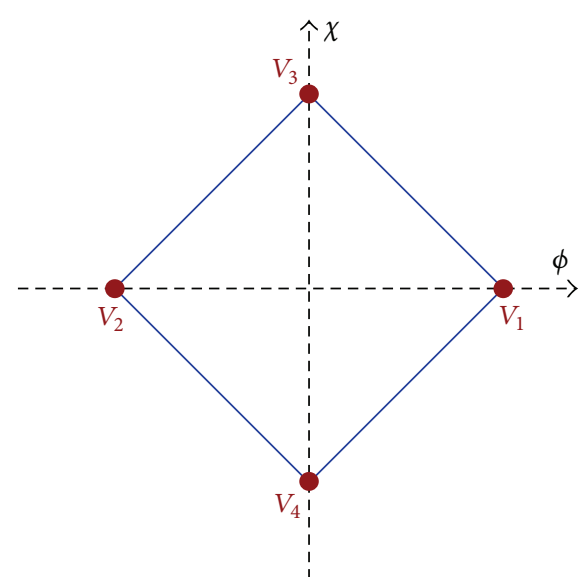

(b)

Figure 2: Graphics of the potential $V(\phi, \chi)$ with $r=1$ and orbits of the kinks (26) in the internal plane.

We can manipulate these equations to get

$$
\phi^{\prime \prime}+\left(4 r \phi-\frac{d f}{d \phi}\right) \phi^{\prime}-4 r \phi f(\phi)=0 .
$$

Choosing $\phi(x)$ as the static solution of a one-field model, such that

$$
\phi^{\prime}=U(\phi), \quad \phi^{\prime \prime}=\frac{d U(\phi)}{d \phi} U(\phi)
$$

we can rewrite (33) as

$$
\frac{d f}{d \phi}+P(\phi) f(\phi)-Q(\phi)=0
$$

where

$$
P(\phi)=\frac{4 r \phi}{U} ; \quad Q(\phi)=4 r \phi+\frac{d U}{d \phi} .
$$

The function $U(\phi)$ is a particular solution of the linear ODE (35), so we can write the general solution in the form

$$
f(\phi)=U(\phi)+C e^{-\int P(\phi) d \phi}
$$

with $C$ being an integration constant. Plugging (37) into (30) we get a one-parameter family of potentials which has a nontrivial two-component kink solution, whose orbit

$$
r \chi^{2}=f(\phi)-U(\phi)
$$

emerges from the use of (32), (34), and (37). Notice that strictly speaking the ODE (35) must be verified only on the kink orbit; in the rest of the internal plane a natural extension of the potential is considered.

We note that the first-order equations (32) support the orbit $\chi(x)=0$, providing us with a second kink solution for 
our model. In this case the static solutions $\phi(x)$ connecting neighbor minima located at the $\phi$-axis are obtained from

$$
\frac{d \phi}{d x}=f(\phi)
$$

The expression $f(\phi)$ coincides with $U(\phi)$ only if the integration constant $C=0$. The general expression of $f(\phi)$ gives rise to a family of two-component field theory models, which admits a two-component kink solution whose first component coincides with the kink associated with $U(\phi)$.

The key step of the procedure appears in (34), and it is inspired by [26]. It works nicely for a variety of choices of $U(\phi)$, and the corresponding models include polynomial and nonpolynomial functions.

3.1. A First Example. To illustrate the previous procedure with concrete examples, let us start considering

$$
U(\phi)=\kappa(a-\phi)(b+\phi),
$$

where $a, b$, and $\kappa$ are real parameters and we obviously assume that $a, b \neq 0$ and $a \neq-b$. We use (37) together with (34) and (36) to obtain

$$
f(\phi)=\kappa(a-\phi)(b+\phi)+C(a-\phi)^{n_{1}}(b+\phi)^{n_{2}},
$$

where

$$
n_{1}=\frac{4 r a}{\kappa(a+b)} ; \quad n_{2}=\frac{4 r b}{\kappa(a+b)} .
$$

This leads to the field potential term

$$
\begin{aligned}
V=\frac{1}{2}[ & \kappa(a-\phi)(b+\phi) \\
& \left.\quad+C(a-\phi)^{n_{1}}(b+\phi)^{n_{2}}-r \chi^{2}\right]^{2}+2 r^{2} \phi^{2} \chi^{2} .
\end{aligned}
$$

Here we have the static solution extracted from (34) for our choice of $U(\phi)$ in (40); it reads

$$
\phi(x)=\frac{a-b}{2} \pm \frac{a+b}{2} \tanh \left(\frac{\kappa(a+b) x}{2}\right),
$$

and from (38) and (41) we obtain

$$
\chi(x)= \pm \sqrt{\frac{C}{r}}(a-\phi(x))^{\left(n_{1} / 2\right)}(b+\phi(x))^{\left(n_{2} / 2\right)} .
$$

Dilatations and translations in the internal space allow us to relocate two vacua placed in the $\phi$-axis at the points $( \pm 1,0)$, such that without loss of generality we can assume that $a=$ 1 and $b=1$. If we restrict ourselves to potentials (43) with a quartic algebraic expression in the fields $\phi$ and $\chi$ we must impose the conditions $4 a r /(a+b) \kappa=1$ and $4 b r /(a+b) \kappa=$ 1 , or equivalently $\kappa=2 r$. In this case we get the family of potentials

$$
V=\frac{1}{2}\left[(2 r+C)\left(1-\phi^{2}\right)-r \chi^{2}\right]^{2}+2 r^{2} \phi^{2} \chi^{2},
$$

where $\mathscr{M}=\left\{v_{1,2}=( \pm 1,0), v_{3,4}=(0, \pm \sqrt{2+C / r})\right\}$ comprises four elements provided that $C>-2 r$. The two-component kinks

$$
\phi(x)=\tanh (2 r x), \quad \chi(x)= \pm \sqrt{\frac{C}{r}} \operatorname{sech}(2 r x)
$$

whose kink orbit is given by $r \chi^{2}=C\left(1-\phi^{2}\right)$ connect the points $v_{3,4}$ and $E_{\mathrm{BPS}}=(4 / 3)(C+2 r)$. The expression (46) can be written as

$$
V=(2 r+C)^{2}\left[\frac{1}{2}\left(1-\phi^{2}-\bar{r} \chi^{2}\right)^{2}+2 \bar{r}^{2} \phi^{2} \chi^{2}\right],
$$

where $\bar{r}=r /(2 r+C)$. A reparametrization of the spatial variable $\bar{x}=(2 r+C) x$ allows us to identify the present example with the potential introduced in the previous section. In this sense if we choose $C=0$ we get the onecomponent topological kink solutions (24). For any other choice of the constant $C$ the solution (47) plays the role of the two-component kink (25). The comparison is straightforward when the constant $2 r+C$ in (46) is unity see Figure 1 . This works as a test for the procedure introduced in this work in a well-known two-field theory model.

If we consider the special case $b=0$ in (40), such that $U=\kappa \phi(a-\phi)$, the use of (37) leads to the function

$$
f(\phi)=\kappa \phi(a-\phi)+C(a-\phi)^{4 r / \kappa},
$$

which generates the field potential

$$
\begin{aligned}
V=\frac{1}{2}[ & \kappa \phi(a-\phi) \\
& \left.+C(a-\phi)^{4 r / \kappa}-r \chi^{2}\right]^{2}+2 r^{2} \phi^{2} \chi^{2} .
\end{aligned}
$$

Because we are interested in quartic potentials in this section, we set $\kappa=2 r$. The previous formula becomes

$$
\begin{aligned}
V= & \frac{1}{2}\left[(a-\phi)(a C+(2 r-C) \phi)-r \chi^{2}\right]^{2} \\
& +2 r^{2} \phi^{2} \chi^{2}
\end{aligned}
$$

whose zeroes are located at $\mathscr{M}=\left\{v_{1}=(a, 0), v_{2}=(-a C /(2 r-\right.$ $\left.C), 0), v_{3,4}=(0, \pm a \sqrt{(C / r)})\right\}$. Equation (38) leads to the kink orbits $\chi= \pm \sqrt{C / r}(a-\phi)$ which connect the points $v_{3,4}$ with $v_{1}$; see Figure 3 . The kink solutions are

$$
\begin{gathered}
\phi=\frac{a}{2}(1+\tanh (\operatorname{ar} x)), \\
\chi= \pm \frac{a}{2} \sqrt{\frac{C}{r}}(1-\tanh (\operatorname{ar} x))
\end{gathered}
$$

whose energy is $E_{\mathrm{BPS}}=a^{3}(C+r) / 3$.

As previously mentioned it is easy to identify the onecomponent kink linking the vacua $v_{1,2}$ for this model. We have

$$
\phi=a+\frac{2 a r}{C-2 r-e^{2 a r x}}, \quad \chi=0,
$$

whose energy is $E_{\mathrm{BPS}}=4 a^{3} r^{3} / 3(C-2 r)^{2}$. 


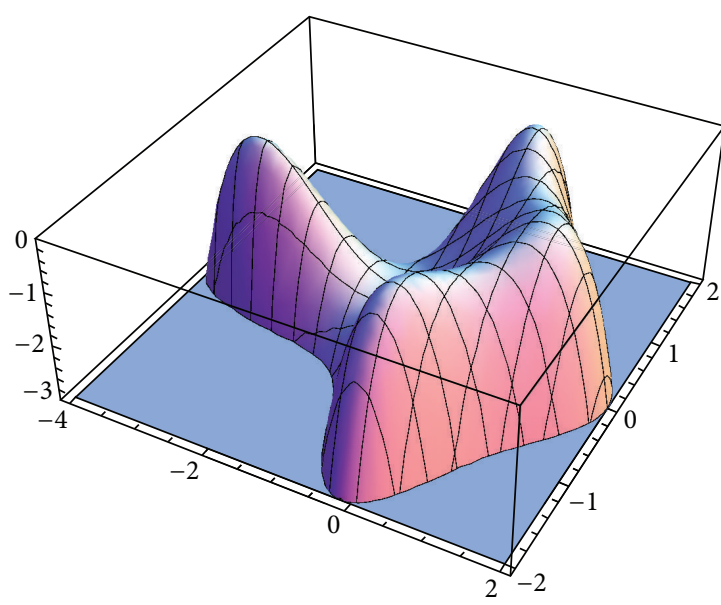

(a)

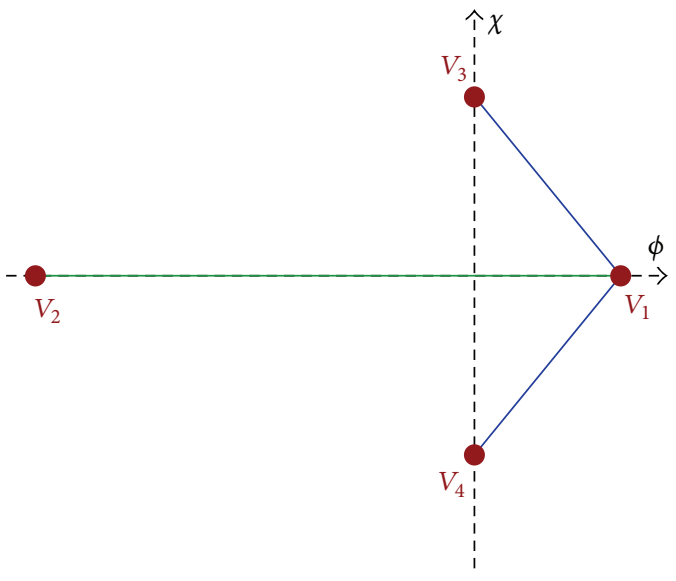

(b)

FIgURe 3: Graphics of the potential $V(\phi, \chi)$ with $r=a=1, C=3 / 2$ and orbits of the kinks (52) and (53) in the internal plane.

Notice that if we consider $r=a=C=\kappa / 2=1$ in (52), we recover the solutions (26) of the test model introduced in the previous section.

The above illustration shows that the procedure works nicely. Thus, below we introduce new families of models using adequate choices of the parameters.

3.1.1. A Family of Models. In the previous section we restrict ourselves to quartic potentials. Here we will introduce expressions of higher degree. Let us choose $a=1, b=1$, and $\kappa=2 r / n$ in (41) and (42) with $n$ as a positive integer. Besides we redefine the coupling constant $r$ as $r=n \bar{r} / 2$. Here we have

$$
f(\phi)=\left(1-\phi^{2}\right)\left(\bar{r}+C\left(1-\phi^{2}\right)^{n-1}\right)
$$

which determines the potentials

$$
\begin{gathered}
V=\frac{1}{2}\left[\left(1-\phi^{2}\right)\left(\bar{r}+C\left(1-\phi^{2}\right)^{n-1}\right)\right. \\
\left.-\frac{n \bar{r}}{2} \chi^{2}\right]^{2}+\frac{n^{2} \bar{r}^{2}}{2} \phi^{2} \chi^{2} .
\end{gathered}
$$

These potentials involve two distinct behaviors depending on $n$ being odd or even.

In the case for $n$ even, there are six degenerate minima at the points $\mathscr{M}_{e}=\left\{v_{1,2}=(0, \pm \sqrt{2(\bar{r}+C) /(n \bar{r})}), v_{3,4}=( \pm 1,0)\right.$, $\left.v_{5,6}=\left( \pm \sqrt{1+(\bar{r} / C)^{1 /(n-1)}}, 0\right)\right\}$ while for $n$ odd there are four degenerate minima; $\mathscr{M}_{o}=\left\{v_{1,2}, v_{3,4}\right\}$. For all models in the above family, we can find the static solutions

$$
\begin{gathered}
\phi(x)= \pm \tanh (\bar{r} x), \\
\chi(x)= \pm \sqrt{\frac{2 C}{n \bar{r}}} \operatorname{sech}^{n}(\bar{r} x),
\end{gathered}
$$

which connect the minima $v_{3}$ and $v_{4}$ by means of the orbit (38) given by the algebraical curve

$$
\bar{r} n \chi^{2}=2 C\left(1-\phi^{2}\right)^{n}
$$

see Figure 4. These solutions carry the energy

$$
E_{\mathrm{BPS}}=\frac{4 \bar{r}}{3}+\frac{\sqrt{\pi} C \Gamma[n+1]}{\Gamma[n+3 / 2]} .
$$

Now for the orbit $\chi(x)=0$ in the BPS sectors connecting neighbor minima at the $\phi$-axis, the solutions are obtained from (39) with $f(\phi)$ given by (41), which may be solved case by case. For $n=1$, we have the solution $\phi(x)= \pm \tanh [(\bar{r}+$ C) $x$ ], and for $n=2$ we get the implicit expression

$$
\frac{(1+\phi)}{(1-\phi)}\left(\frac{\sqrt{1+(\bar{r} / C)}-\phi}{\sqrt{1+(\bar{r} / C)}+\phi}\right)^{\sqrt{C /(C+\bar{r})}}=e^{2 \bar{r} x}
$$

and so on for other values of $n$. It is remarkable that we can obtain the explicit expression of the two-component kinks (56) for any value of $n$ but not for the one-component kinks.

3.1.2. Another Family of Models. Here we take $b=0, \kappa=4 r / n$, $a>0$, and integer $n>0$ and we redefine the coupling constant $r$ as $r=(\bar{r} n) / 4$. From (41) we have

$$
f(\phi)=(a-\phi)\left(\bar{r} \phi+C(a-\phi)^{n-1}\right),
$$

which generate a family of models whose potentials have up to $n+2$ minima: two minima at $v_{1,2}=\left(0, \pm 2 \sqrt{\left(a^{n} C\right) /(\bar{r} n)}\right)$, one at $v_{3}=(a, 0)$, and up to $n-1$ minima (for $\left.n>1\right)$ coming from the condition $g(\phi)=\bar{r} \phi+C(a-\phi)^{n-1}=0$. 


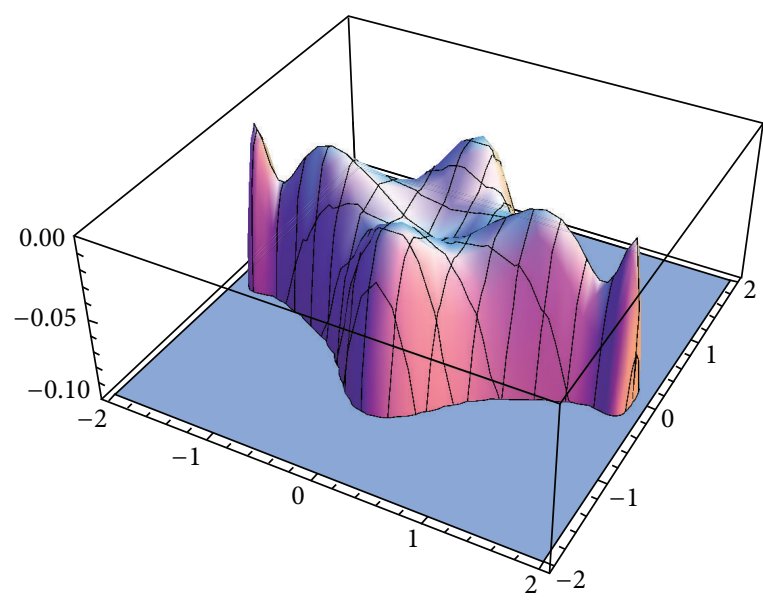

(a)

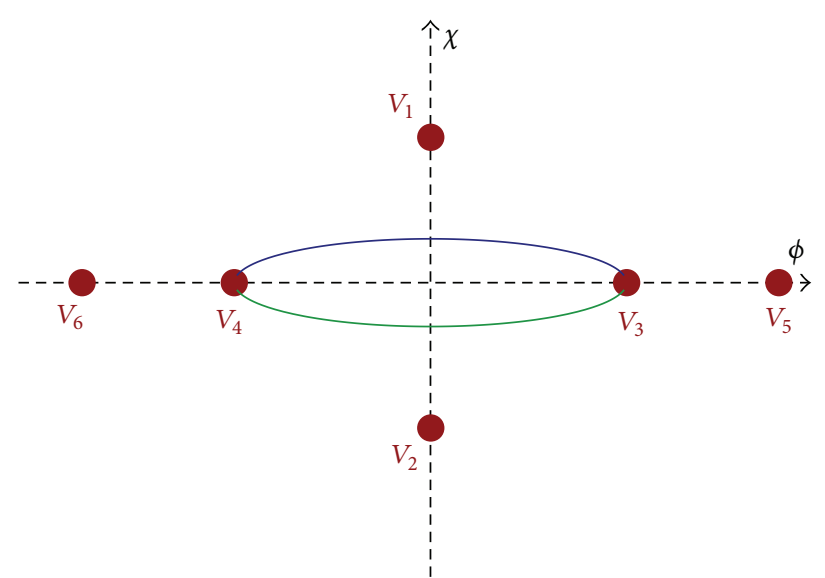

(b)

FIGURE 4: Graphics of the potential $V(\phi, \chi)$ with $n=4$ and orbits of the kinks (56) in the internal plane.

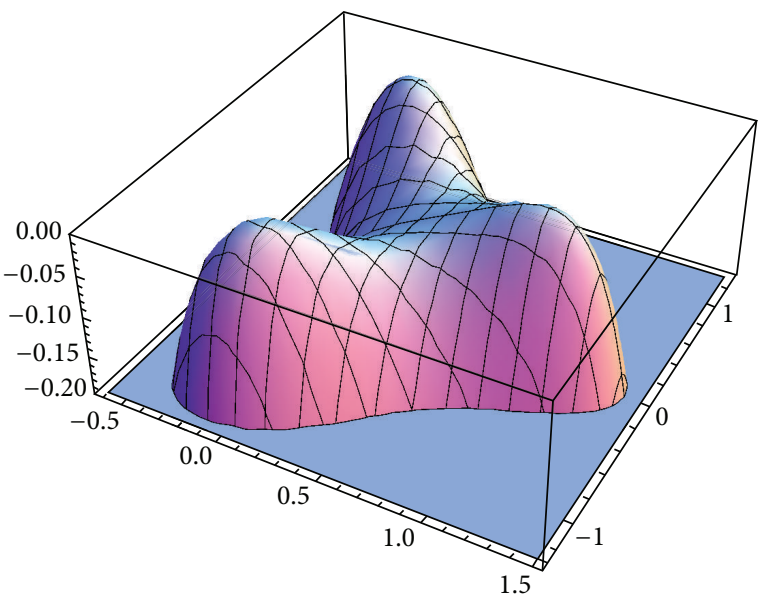

(a)

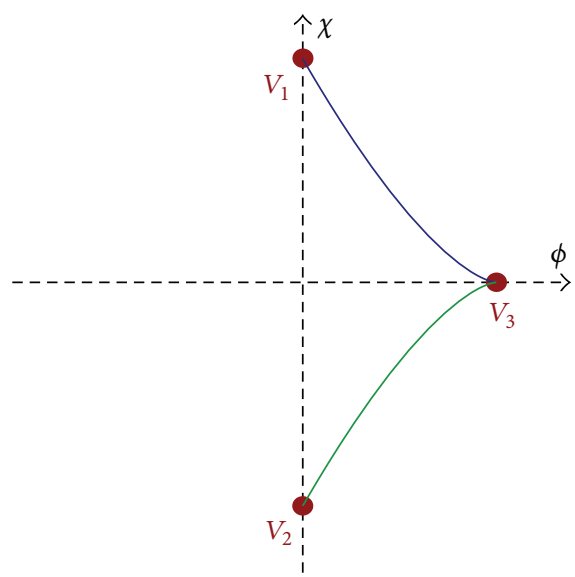

(b)

FIgURE 5: Graphics of the potential $V(\phi, \chi)$ with $n=4$ and orbits of the kinks (61) in the internal plane.

For all models of this family, we get from (38), (44), and (40) in the sector connecting the minima $v_{1,2}$ and $v_{3}$ the static solutions

$$
\begin{gathered}
\phi(x)=\frac{a}{2}\left(1 \pm \tanh \left(\frac{a \bar{r} x}{2}\right)\right), \\
\chi(x)= \pm 2^{1-(n / 2)} \sqrt{\frac{c}{\bar{r} n}} a^{n / 2}\left(1-\tanh \left(\frac{a \bar{r} x}{2}\right)\right)^{n / 2},
\end{gathered}
$$

whose energy is given by $E_{\mathrm{BPS}}=\left(\bar{r} a^{3} / 6\right)+\left(C a^{n+1} /(n+1)\right)$; see Figure 5.

\section{Nonpolynomial Models}

Let us now move on to the case of nonpolynomial potentials. Here we consider

$$
W(\phi, \chi)=\int^{\phi} f(y) d y-r \phi \sin \chi
$$

such that the field potential is

$$
V=\frac{1}{2}\left[(f(\phi)-r \sin \chi)^{2}+r^{2} \phi^{2} \cos ^{2} \chi\right],
$$

which is a periodic function in the variable $\chi$ as illustrated in Figure 6. The set of zeroes is given by $\mathscr{M}=\left\{v_{1, m_{1}}, v_{2, m_{2}}\right.$, $\left.v_{i+2, m_{3}}\right\}$, where

$$
\begin{gathered}
v_{1, m_{1}}=\left(0, \arcsin \frac{f(0)}{r}+2 \pi m_{2}\right), \\
v_{2, m_{2}}=\left(0,-\arcsin \frac{f(0)}{r}+\pi\left(2 m_{3}+1\right)\right), \\
v_{i+2, m_{3}}=\left(\phi^{(i)}, \pi\left(m_{1}+\frac{1}{2}\right)\right),
\end{gathered}
$$




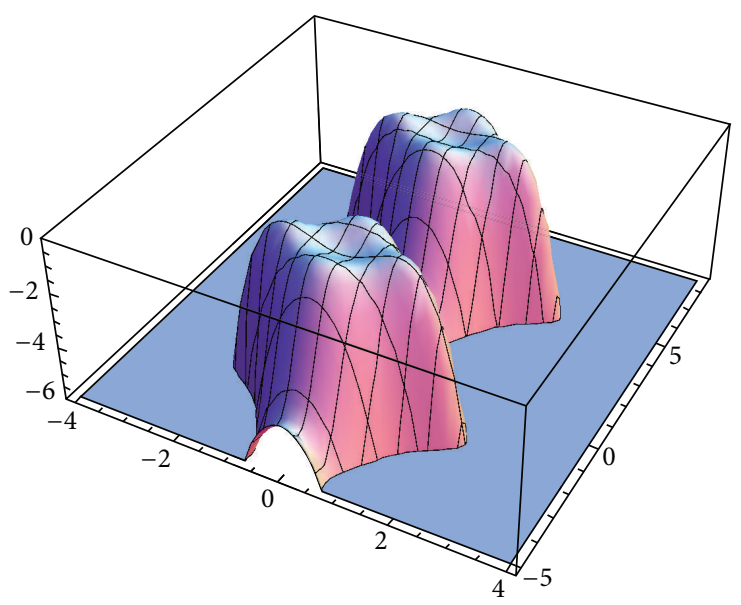

(a)

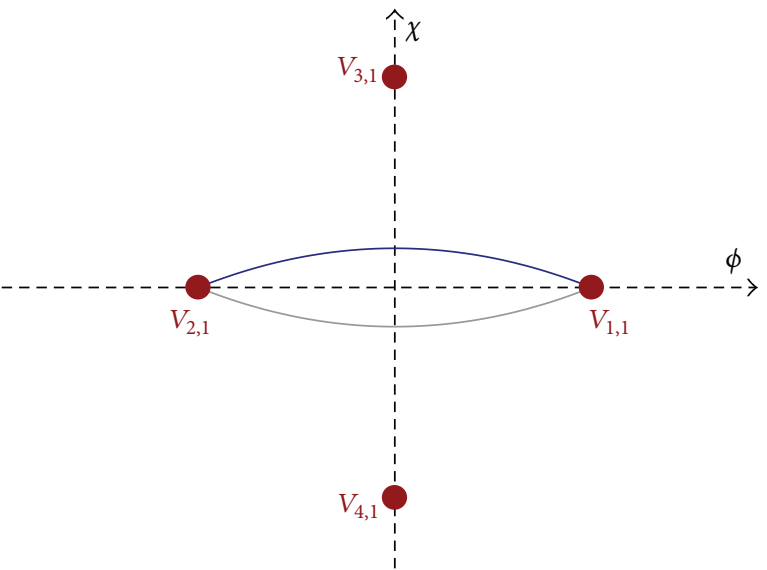

(b)

FIgURE 6: Graphics of the potential $V(\phi, \chi)$ and orbits of the kinks (76) in the internal plane for $n=2$.

where $\phi^{(i)}$ are the roots of the function $f(\phi)-(-1)^{n} r$, and $m_{i} \in \mathbb{Z}$. In this case, the static solutions are obtained from the first-order equations

$$
\phi^{\prime}=f(\phi)-r \sin \chi, \quad \chi^{\prime}=-r \phi \cos \chi .
$$

We can manipulate these equations to get

$$
\phi^{\prime \prime}+\phi \phi^{\prime 2}-\left(2 \phi f(\phi)+\frac{d f}{d \phi}\right) \phi^{\prime}-\phi\left(r^{2}-f^{2}(\phi)\right)=0 .
$$

Again, choosing $\phi(x)$ satisfying (34) we can rewrite the above equation as

$$
\frac{d f}{d \phi}+P(\phi) f(\phi)-R(\phi) f^{2}(\phi)-Q(\phi)=0,
$$

where

$$
\begin{gathered}
P(\phi)=2 \phi, \quad R(\phi)=\frac{\phi}{U}, \\
Q(\phi)=\frac{d U}{d \phi}+\phi U-\frac{r^{2} \phi}{U} .
\end{gathered}
$$

The general solution is given by

$$
f(\phi)=U(\phi)+r \tanh \left[r\left(C-\int \frac{\phi d \phi}{U(\phi)}\right)\right] .
$$

Then, choosing $U(\phi)$ we have the constraint

$$
r \sin \chi=f(\phi)-U(\phi)
$$

for the field $\chi$.

Also, the first-order equations (65) support the orbit $\chi(x)= \pm(2 n-1) \pi / 2, n=0,1,2, \ldots$. In this case, the static solutions $\phi(x)$, connecting neighbor minima located at the $\phi$-axis, are obtained from the equation

$$
\frac{d \phi}{d x}=f(\phi)+(-1)^{n} r
$$

Let us now illustrate the above procedure with an example. We start using

$$
U(\phi)=\kappa(a-\phi)(b+\phi),
$$

where $a, b, \kappa$ are real parameters. By employing (69) we obtain

$$
\begin{aligned}
(\phi)= & U(\phi) \\
& +r \frac{C^{2}(a-\phi)^{2 a r / \kappa(a+b)}(b+\phi)^{2 b r / \kappa(a+b)}-1}{C^{2}(a-\phi)^{2 a r / \kappa(a+b)}(b+\phi)^{2 b r / \kappa(a+b)}+1} .
\end{aligned}
$$

Again we restrict ourselves to cases where the exponents in the previous expression are even integers, such that we impose that $\operatorname{ar} / \kappa(a+b)=n$ with $n \in \mathbb{N}$, for instance, by choosing $\kappa=a r / n(a+b)$. In this case

$$
\begin{aligned}
f(\phi)= & U(\phi) \\
& +r \frac{C^{2}(a-\phi)^{2 n}(b+\phi)^{2 n}-1}{C^{2}(a-\phi)^{2 n}(b+\phi)^{2 n}+1} .
\end{aligned}
$$

The field potential term is obtained by plugging (74) into (63). In spite of the complexity of this expression the kink solution is written by the expression (44) and from (70) as follows:

$$
\chi=\arcsin \frac{C^{2}(a-\phi(x))^{2 n}(b+\phi(x))^{2 n}-1}{C^{2}(a-\phi(x))^{2 n}(b+\phi(x))^{2 n}+1} .
$$

For example for $a=b=1$ we get

$$
\begin{gathered}
\phi=\tanh \frac{r x}{2 n}, \\
\chi=\arcsin \left(1-\frac{2}{1+C^{2} \operatorname{sech}^{2 n}(r x / 2 n)}\right),
\end{gathered}
$$

whose orbit is displayed in Figure 6 for the case $n=2$. 


\section{Final Comments}

In this work we proposed a new method to construct and solve models described by two real scalar fields. The procedure is simple, inspired by the approach introduced in [26], and it works for the construction of polynomial and nonpolynomial models.

To illustrate the procedure, we studied several examples, which show how efficient the method is, in order to construct new two-field models with nontrivial two-component kink solutions. We note that the method starts with $W$, the superpotential, so all the models we construct lead to first-order differential equations, which solve the equations of motion. In this sense, all the solutions we found are BPS states, and they are classically or linearly stable, as proved before in [27].

A relevant feature of the procedure is that it is different from the deformation procedure involving two-field models, and it is very simple to be applied in investigations based on two real scalar fields. An issue which deserves further examination concerns the extension of the method to three or more real scalar fields. This is under investigation, and we hope to report the new results in a separate work.

\section{Acknowledgment}

The authors would like to thank CAPES, CNPq, and FAPESP for partial financial support.

\section{References}

[1] A. Vilenkim and E. P. S. Shellard, Cosmic Strings and other Topological Defects, Cambridge University Press, Cambridge, UK, 1994.

[2] N. Manton and P. Sutcliffe, Topological Solitons, Cambridge University Press, Cambridge, UK, 2004.

[3] G. B. Whitham, Linear and Nonlinear Waves, Wiley, New York, NY, USA, 1974.

[4] D. Walgraef, Spacio-Temporal Pattern Formation, Springer, Berlin, Germany, 1981.

[5] G. Basar and G. V. Dunne, "Self-consistent crystalline condensate in chiral Gross-Neveu and Bogoliubov-de Gennes systems," Physical Review Letters, vol. 100, no. 20, Article ID 200404, 4 pages, 2008.

[6] A. Alonso-Izquierdo, M. A. G. Leon, and J. M. Guilarte, "Kinks in a nonlinear massive sigma model," Physical Review Letters, vol. 101, no. 13, Article ID 131602, 2008.

[7] P. O. Jubert, R. Allenspach, and A. Bischof, "Magnetic domain walls in constrained geometries," Physical Review B, vol. 69, no. 22, Article ID 220410R, 4 pages, 2004.

[8] A. Vanhaverbeke, A. Bischof, and R. Allenspach, "Control of domain wall polarity by current pulses," Physical Review Letters, vol. 101, no. 10, Article ID 107202, 4 pages, 2008.

[9] J. Belmonte-Beitia, V. M. Perez-Garcia, V. Vekslerchik, and V. V. Konotop, "Localized nonlinear waves in systems with timeand space-modulated nonlinearities," Physical Review Letters, vol. 100, no. 16, Article ID 164102, 4 pages, 2008.

[10] A. T. Avelar, D. Bazeia, and W. B. Cardoso, "Solitons with cubic and quintic nonlinearities modulated in space and time," Physical Review E, vol. 79, no. 2, Article ID 025602R, 4 pages, 2009.
[11] J. Casahorrán, "Quantum-mechanical tunneling: differential operators, zeta-functions and determinants," Fortschritte der Physik, vol. 50, no. 3-4, pp. 405-424, 2002.

[12] M. Bordag and A. Yurov, "Spontaneous symmetry breaking and reflectionless scattering data," Physical Review D, vol. 67, no. 2, Article ID 025003, 9 pages, 2003.

[13] A. Alonso-Izquierdo and J. M. Guilarte, "On a family of $(1+$ 1)-dimensional scalar field theory models: kinks, stability, oneloop mass shifts," Annals of Physics, vol. 327, no. 9, pp. 2251-2274, 2012.

[14] R. Rajaraman, Solitons and Instantons. An Introduction to Solitons and Instantons in Quantum Field Theory, North-Holland Publishing, Amsterdam, The Netherlands, 1987.

[15] A. Alonso Izquierdo and J. M. Guilarte, "Generalized MSTB models: structure and kink varieties," Physica D, vol. 237, no. 24, pp. 3263-3291, 2008.

[16] A. Alonso-Izquierdo and J. M. Guilarte, "Composite solitary waves in three-component scalar field theory: three-body lowenergy scattering," Physica D, vol. 220, no. 1, pp. 31-53, 2006.

[17] M. K. Prasad and C. M. Sommerfeld, "Exact classical solution for the 't Hooft monopole and the Julia-Zee dyon," Physical Review Letters, vol. 35, no. 12, p. 760, 1975.

[18] E. B. Bolgomol'nyi, “The stability of classical solutions," Soviet Journal of Nuclear Physics, vol. 24, no. 4, pp. 449-454, 1976.

[19] B. Chibisov and M. Shifman, "BPS-saturated walls in supersymmetric theories," Physical Review D, vol. 56, no. 12, pp. 79908013, 1997, Erratum in: Physical Review D, vol. 58, Article ID 109901, 1998.

[20] M. A. Shifman and M. B. Voloshin, "Degenerate domain wall solutions in supersymmetric theories," Physical Review D, vol. 57, pp. 2590-2598, 1998.

[21] D. Bazeia, M. J. dos Santos, and R. F. Ribeiro, "Solitons in systems of coupled scalar fields," Physics Letters A, vol. 208, no. 1-2, pp. 84-88, 1995.

[22] D. Bazeia, J. R. S. Nascimento, R. F. Ribeiro, and D. Toledo, "Soliton stability in systems of two real scalar fields," Journal of Physics A, vol. 30, no. 23, pp. 8157-8166, 1997.

[23] D. Bazeia and F. A. Brito, "Bags, junctions, and networks of BPS and non-BPS defects," Physical Review D, vol. 61, no. 10, Article ID 105019, 16 pages, 2000.

[24] A. Alonso-Izquierdo, M. A. G. Leon, and J. M. Guilarte, "The Kink variety in systems of two coupled scalar fields in two spacetime dimensions," Physical Review D, vol. 65, Article ID 085012, 2002.

[25] A. Alonso-Izquierdo, M. A. G. Leon, J. M. Guilarte, and M. D. Mayado, "Adiabatic motion of two-component BPS kinks," Physical Review D, vol. 66, no. 10, Article ID 105022, 9 pages, 2002.

[26] D. Bazeia, A. Das, L. Losano, and M. J. dos Santos, "Traveling wave solutions of nonlinear partial differential equations," Applied Mathematics Letters, vol. 23, no. 6, pp. 681-686, 2010.

[27] D. Bazeia and M. M. Santos, "Classical stability of solitons in systems of coupled scalar fields," Physics Letters A, vol. 217, no. 1, pp. 28-30, 1996. 

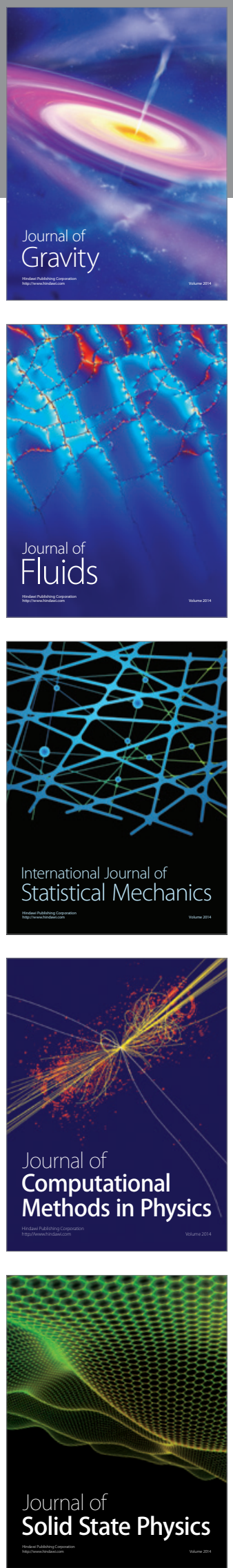

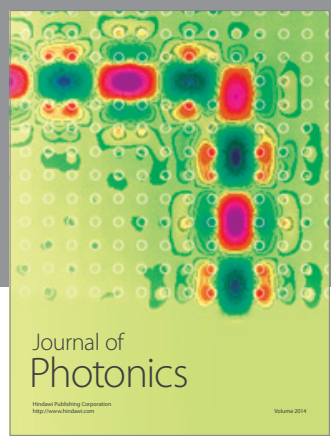

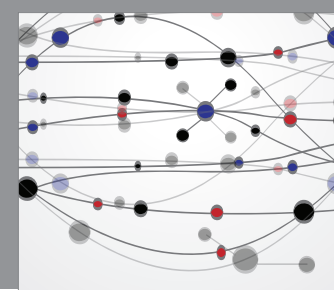

The Scientific World Journal

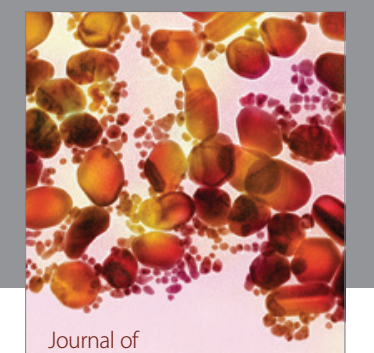

Soft Matter
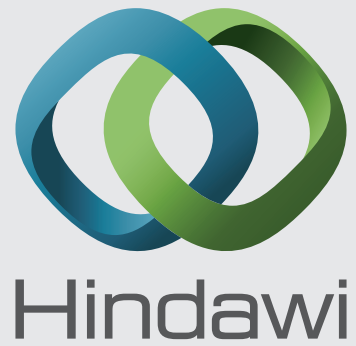

Submit your manuscripts at

http://www.hindawi.com
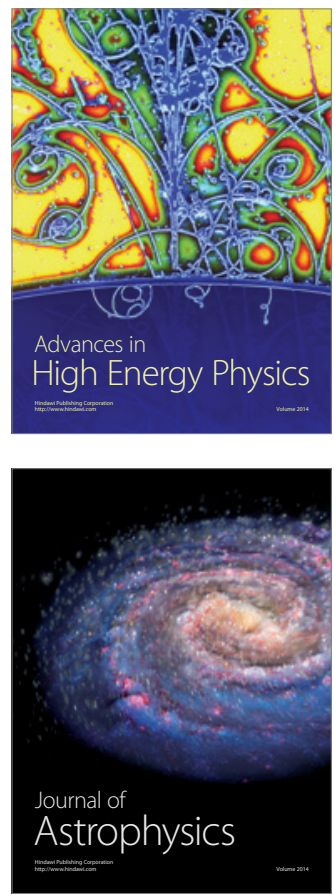
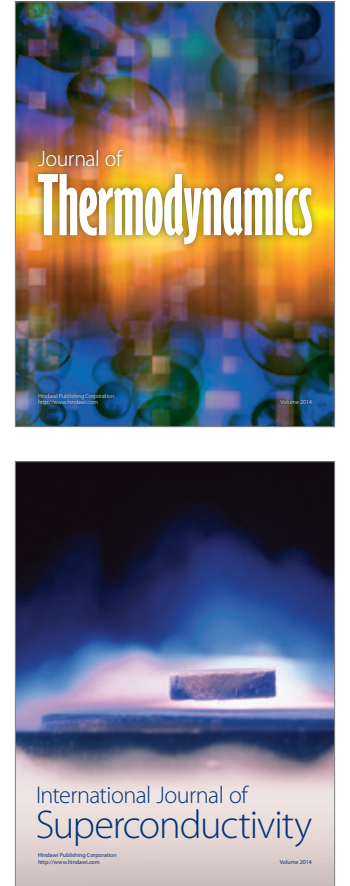
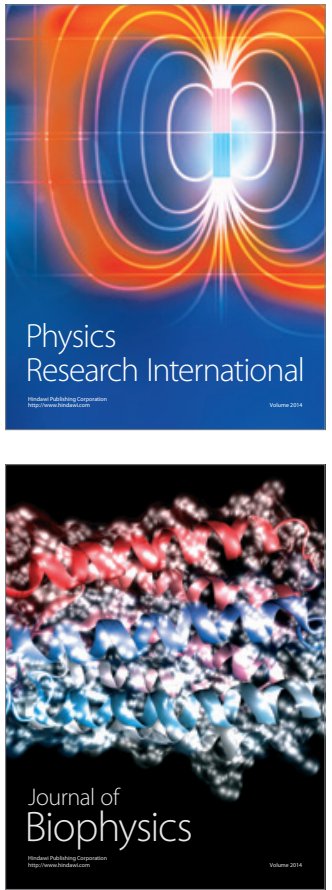
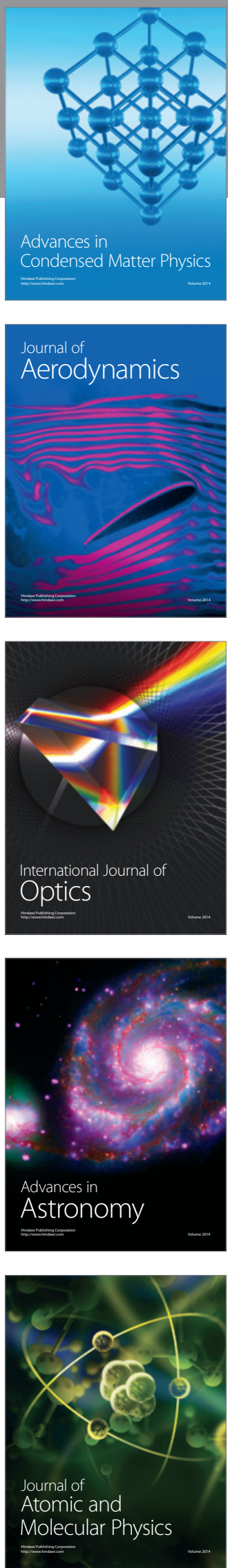